\title{
Innerschulische Strategien der Verarbeitung von Resultaten externer Evaluationen. Eine fallvergleichende Studie zu obligatorischen Schulen in der Schweiz
}

\section{Sara Mahler, Carsten Quesel, Andrea Höchli und Kirsten Schweinberger}

Externe Evaluationen sollen Aufschluss über die Schulqualität geben und zur Schulentwicklung beitragen. Der Beitrag behandelt die Frage nach den Strategien der innerschulischen Verarbeitung von Resultaten externer Schulevaluation anhand von qualitativen Fallstudien in vier Schweizer Kantonen. In der längsschnittlichen Begleitung treten vier Verarbeitungsstrategien hervor, die sich zum einen hinsichtlich der Steuerungsverfahren und zum anderen hinsichtlich der Gegenstandsbereiche der getroffenen Massnahmen unterscheiden. Die Befunde weisen darauf hin, dass die gewählten Strategien von den Themen abhängig sind, die im Evaluationsbericht im Vordergrund stehen. Je grösser die Bedeutung pädagogischer Fragen, desto stärker ist das Kollegium in den Prozess der Ergebnisverarbeitung involviert.

Gegenstand dieses Artikels sind Verarbeitungsstrategien, die die Schulen nach der Rückmeldung der Ergebnisse der externen Evaluation wählen und in ersten Schritten umsetzen. Grundlage bilden 14 qualitative Fallstudien aus vier verschiedenen Schweizer Kantonen, die sich hinsichtlich der lokalen Gestaltungsautonomie unterscheiden ${ }^{1}$. Der Untersuchungszeitraum erstreckt sich auf das Jahr nach der Rückmeldung der Ergebnisse der externen Schulevaluation. Der Beitrag zielt darauf $\mathrm{ab}$, Bedingungen und Varianten evidenzbasierter Schulentwicklung herauszuarbeiten.

Die Implementierung von Prinzipien des New Public Management haben im Bildungssystem zu Varianten einer outputorientierten Neuen Steuerung geführt (Altrichter \& Maag Merki, 2010). Die Schule wird dabei als «Leistungserbringer im öffentlichen Raum» (Quesel, Näpfli, Buser \& van der Heiden, 2015, S. 240) definiert, die im Rahmen von Leistungsaufträgen über interne Gestaltungsfreiheit verfügen, jedoch dem politisch-administrativen System rechenschaftspflichtig sind. Die Überprüfung erfolgt unter anderem in Form externer Evaluationen. Dabei wird die Qualität der Einzelschule anhand von systematisch und regelmässig erhobenen Daten aus einer Perspektive der professionellen Fremdbeobachtung überprüft und beurteilt (Dedering, 2012). Im deutschen Sprachraum richtet sich der Fokus auf die Überprüfung der Prozessqualitäten, 
von denen erwartet wird, dass sie einen direkten oder indirekten Einfluss auf die Lernergebnisse haben (Böttger-Beer, Vaccaro \& Koch, 2010).

Landwehr (2011) unterscheidet vier verschiedene Wirkbereiche externer Evaluationen, die sich wechselseitig beeinflussen und bedingen: die Wissensgewinnung, die Rechenschaftslegung, die Normdurchsetzung sowie die Schulentwicklung. Die vorliegende Studie konzentriert sich auf den Aspekt der Entwicklungswirksamkeit, der "vermutlich die grundlegendste und wichtigste Erwartungshaltung» (ebd., S. 48) betrifft, mit der die externe Schulevaluation konfrontiert ist: Das Sammeln und Auswerten von Daten soll zu Verbesserungen führen.

Fraglich ist allerdings, ob sich solche Verbesserungen gleichsam automatisch aus der Diagnose ergeben. Wer einen solchen Automatismus unterstellt, lässt sich von der Prämisse leiten, dass Schulleitungen und Lehrpersonen sich beim Auftreten von Ist-Soll-Diskrepanzen dem Appell von Anregungen oder Empfehlungen nicht entziehen können, die auf die Überwindung der nachgewiesenen Defizite abzielen (Dedering \& Tillmann, 2012; Landwehr, 2011); die Beachtung des Appells sollte dann über kurz oder lang zu verbesserten Lernleistungen führen (Pietsch, van de Ham \& Köller, 2015). Mithin wird erwartet, dass die Übermittlung von Evidenzen aus der externen professionellen Beobachterperspektive kraft des überzeugenden Arguments zu einem effektiven und effizienten Engagement für die Schul- und Unterrichtsqualität führt (Böttcher, Dicke \& Hogrebe, 2010).

Dieser Erwartungshaltung widersprechen Forschungsbefunde, die darauf hindeuten, dass die neu eingeführten Instrumente bislang wenig Spuren im Bereich der Schulentwicklung hinterlassen haben (Altrichter \& Maag Merki, 2010; Wacker, Maier \& Wissinger, 2012) und gravierende nichtintendierte Effekte auslösen (Bellmann \& Weiss, 2009). Bislang fehlen starke empirische Evidenzen, die Effekte externer Evaluationen in Bezug auf den Unterricht und die Lernleistungen nachweisen (Ehren \& Visscher, 2006; Pietsch et al. 2015). Das liegt auch daran, dass die meisten der bislang eingesetzten Forschungsdesigns sich auf die Ermittlung der Akzeptanz von Evaluationen oder auf das Auszählen von ergriffenen Massnahmen beschränken und von daher wenig geeignet sind, um tatsächlich etwas über kausale Zusammenhänge auszusagen (Husfeldt, 2011).

\section{Wirkmodelle externer Evaluationen}

Ehren und Visscher (2006) führen in ihrem Wirkmodell (Abb. 1) Bedingungen und Faktoren auf, die den Prozess von der externen Evaluation bis zum Output ergriffener Massnahmen nachzeichnen. 


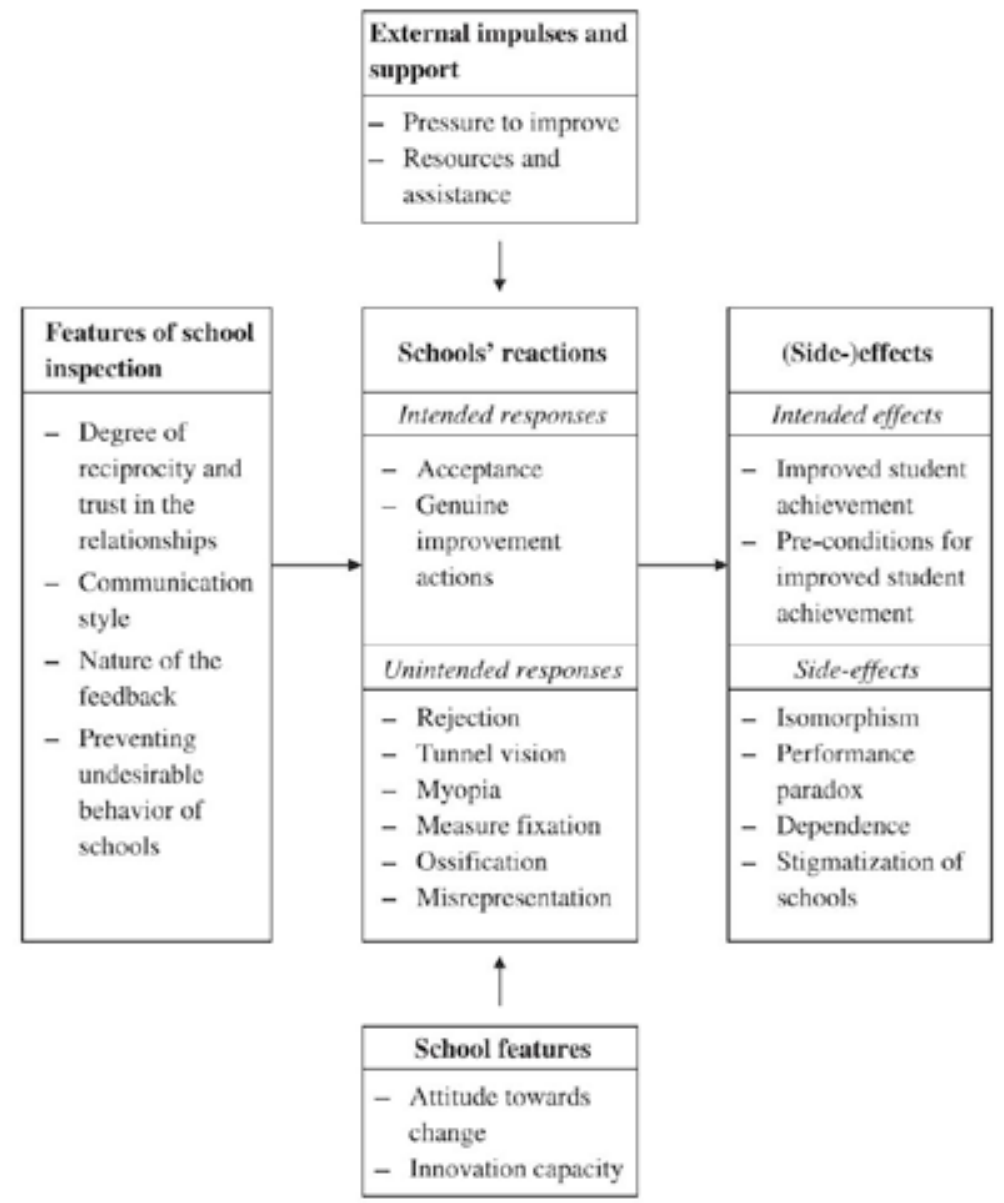

Abbildung 1: Wirkmodell von Ehren und Visscher (2006)

Das Modell baut auf dem CIPO-Prozessmodell der Schuleffektivitätsforschung (Ditton, 2002; Scheerens \& Bosker, 1997) auf, wobei zwischen dem Input in Form der externen Evaluation, der Verarbeitung der Ergebnisse und dem Output in Form der Gestaltung von lernförderlichen Bedingungen und Lernleistungen sowie Nebenwirkungen unterschieden wird.

Die Betrachtung des Modells macht deutlich, dass Ehren und Visscher (2006) den Schritt der schulischen Reaktion nicht weiter aufschlüsseln (Böttger-Beer et al. 2010). Im Hinblick auf diese Reaktionen beschreiben Stamm (2003) sowie Wurster und Gärtner (2013) verschiedene Nutzungstypen, die sich auf die Art der Ergebnisse und deren Wahrnehmung beziehen. Die Typologie von Stamm unterscheidet zwischen Blockade, Innovation, Alibi und Reaktion. Die Typologie 
von Wurster und Gärtner zwischen aktiven Schulen, reaktiven Schulen, aktiv unzufriedenen Schulen, (selbst-)zufriedenen Schulen und unzufriedenen Schulen. Bellmann, Duzevic, Kirchhoff und Schweizer (2014) unterteilen Reaktionen und Interpretationen schulischer Akteure im Kontext outputorientierter Steuerung mit Blick auf deren Nebenfolgen. Die von ihnen bezeichneten Typen unterscheiden sich zum einen im Hinblick auf die Akzeptanz der Praxis dieser Steuerung und zum anderen im Hinblick auf die Sensibilität für deren unbeabsichtigte Auswirkungen. Professionalität erscheint in diesem Blickwinkel als eine Ressource, sich Zumutungen der Outputorientierung aus pädagogischen Erwägungen zu widersetzen.

Diese Typologien fokussieren entweder auf die Schule als kollektiven Akteur oder auf individuelle Akteure wie Lehr- und Leitungspersonen. Der vorliegende Beitrag zielt darauf ab, die Kooperation innerhalb der Schule bei der Interpretation und der Verarbeitung von Resultaten externer Evaluationen ins Zentrum der Aufmerksamkeit zu rücken.

Um diese Aspekte zu erfassen, ist es sinnvoll, den Bezugsrahmen für Gelingensbedingungen von Schulentwicklungsprozessen von Reezigt und Creemers (2005) hinzu zu ziehen. Die Autoren unterteilen den Entwicklungsprozess in die Phasen der Diagnose, der Affirmation des Entwicklungsbedarfs, die Planung der Aktivitäten, der Umsetzung des Entwicklungsplans und der Evaluation. Diese Prozessschritte können sich überlappen sowie wiederholen. Im Fall von externen Evaluationen wird die Diagnose von aussen vorgenommen, sodass der innerschulische Entwicklungsprozess mit der Affirmation des Entwicklungsbedarfs beginnt. Ein Vergleich dieser Entwicklungsschritte mit Modellen der Organisationsentwicklung (French \& Bell, 1990) sowie dem Institutionellen Schulentwicklungsprozess von Dalin, Rolff und Buchen (1996) offenbart ein hohes Mass an Übereinstimmung. Dementsprechend folgt dieser Beitrag der Einteilung des Verarbeitungsprozesses in die Phasen des Problemnachvollzugs, der Planung der Aktivitäten, der Umsetzung des Entwicklungsplans sowie der Evaluation der Massnahmen für die Strukturierung des Schulentwicklungsprozess, der sich auf die Organisations-, Personal- und Unterrichtsentwicklung erstreckt (Rolff, 2007).

\section{Nutzungsstrategien}

Weiss (1998a) verweist darauf, dass die Bejahung von Evaluationsresultaten nicht garantiert, dass die Evidenzen für Weiterentwicklungen genutzt werden. Machtverhältnisse und implizite Regeln der Organisation können einer solcher Nutzung ebenso im Weg stehen wie Ressourcenknappheit. Bei genauem Hinsehen erweist es sich zudem als wichtig, den Begriff der Nutzung zu differenzieren. King und Pechman (1984) sowie Weiss (1998b) unterteilen den Umgang mit Evaluationsergebnissen in drei Nutzungsformen. 
1.) Instrumental Use: Die Evidenzen der externen Evaluation werden relativ direkt für Massnahmen genutzt, die darauf abzielen, dass die Organisation die von innen oder aussen definierten Zwecke besser erreicht.

2.) Conceptual Use: Die Ergebnisse der Evaluation werden als Orientierungshilfe benutzt und dienen dazu, den eigenen Interpretationsrahmen zu überprüfen und möglicherweise zu verändern.

3.) Symbolic Use: Die Evaluationsergebnisse werden demonstrativ dafür genutzt, die Legitimität der bestehenden Praxis zu unterstreichen; mitunter werden Schattenseiten dieser Praxis durch window dressing verhüllt, zuweilen wird Aktivität durch formalistische Anpassungen vorgetäuscht.

Empirisch sind die drei Nutzungsformen nicht immer eindeutig voneinander abgrenzbar, da die Ziele von Organisationen mehrdeutig sein und Evaluationsresultate aus verschiedenen Perspektiven interpretiert und mit verschiedenen Absichten verwendet werden können. Wenn Akteure in komplexen Organisationen verschiedene Präferenzen haben, führt die Auswertung von Informationen und die Abwägung von Alternativen nicht unbedingt zu einem klaren Befund, der den Weg einer optimalen Entscheidung vorzeichnet (Weiss, 1998a). Es kann dazu kommen, dass sich bei der Verarbeitung von Evaluationsergebnissen talk und action entkoppeln (Brunsson, 2002): Die Papiere verheissen eine weitreichende Veränderungen und erwecken den Anschein des Wandels, obwohl die Praxis in den gewohnten Bahnen verläuft.

\section{Forschungsfragen und Arbeitshypothesen}

Die vorliegende Studie geht den Fragen nach, wie sich die schulischen Entwicklungsaktivitäten in Folge externer Evaluationen gestalten und wie sich unterschiedliche Rahmenbedingungen auf diesen Prozess auswirken. Dabei stehen die Rezeption der Evaluationsergebnisse und die Ableitung von Massnahmen im Zentrum.

Die unterschiedlichen Rahmenbedingungen sind im Fall des schweizerischen Bildungssystems eine Folge der föderalen Vielfalt: Die Kantone reglementieren die Schritte für die Verarbeitung von Evaluationsergebnissen unterschiedlich stark (Mahler \& Quesel, 2015). Dabei zeigen sich zwei verschiedene Pole: Den einen Pol markieren Ordnungen, in denen die Schulen explizite Empfehlungen erhalten, die sie zumindest teilweise in Form von Massnahmen aufgreifen müssen. Die Massnahmenplanungen sowie die Umsetzung werden dabei durch den Kanton eng begleitet und kontrolliert. Am anderen Pol finden sich Ordnungen, in denen die Schulen selbst entscheiden, ob sie Empfehlungen erhalten und wie sie mit dem Bericht verfahren, wobei in diesen Fällen keine kantonale Begleitung oder Kontrolle stattfindet.

Zur kantonalen Dimension der Regulierungstiefe kommt die lokale Dimension als zweite Achse hinzu: Hier wird zwischen kleineren und grösseren 
Schulen unterschieden, wobei die Annahme lautet, dass mit der Grösse der Schulen auch die Komplexität der Interaktionen bei der Verarbeitung der Evaluationsergebnisse zunimmt.

Die zwei Variablen der Schulgrösse und der kantonalen Regulierung ergeben eine Vierfelder-Matrix (Abb. 2), auf die sich vier folgenden Arbeitshypothesen beziehen.

Arbeitshypothese 1: Verarbeitungsstrategien an Schulen mit hoch regulierten Vorgaben ähneln einander, da der schulische Autonomiespielraum relativ stark eingeschränkt ist.

Arbeitshypothese 2: Verarbeitungsstrategien an Schulen mit gering regulierten Vorgaben sind stärker durch das Führungsverständnis und das Führungsverhalten der Schulleitung geprägt und weisen daher eine grössere Breite auf.

Arbeitshypothese 3: An kleinen Schulen ist die Partizipation der Kollegien aufgrund der stärker ausgeprägten sozialen Nähe höher als an grösseren Schulen. Arbeitshypothese 4: An grossen Schulen in hoch regulierten Evaluationssystemen zeigt sich die geringste Partizipation des Kollegiums.

Eine weitere Arbeitshypothese ergibt sich im Hinblick auf die inhaltlichen Aussagen der Evaluationsberichte. Ehren, Altrichter, McNamara und O'Hara (2013) betonen, dass Feedback nicht automatisch zu einer Entwicklung führt. Inhalt, Format und Kommunikationsstil bestimmen, inwieweit die Rückmeldungen von Schulführungen und Lehrpersonen verarbeitet werden. Feststellungen und Empfehlungen können sich auf formale Merkmale der Organisationsstruktur der Schulen oder auf Aspekte der pädagogischen Arbeit beziehen. Je nachdem, welche Schwerpunkte der Evaluationsbericht setzt, ergeben sich unterschiedliche Perspektiven für die Partizipation des Kollegiums. Verhaeghe, Vanhoof, Valcke und Van Petegem (2009) stellten fest, dass Lehrpersonen Feedback auf Schüler-/Schülerinnenebene bevorzugen, während politische Entscheidungsträger aggregierte Informationen auf Schulebene favorisieren. $\mathrm{Zu}$ vermuten ist, dass Schulleitungspersonen diese Diskrepanz feststellen und darauf mit unterschiedlichen Partizipationsmöglichkeiten für das Kollegium reagieren. Aus diesen Überlegungen heraus ergibt sich die Arbeitshypothese 5: Je grösser das Gewicht pädagogischer Fragen im Evaluationsbericht, desto stärker ist das Kollegium in die Ergebnisverarbeitung involviert. 
kleine Schulen

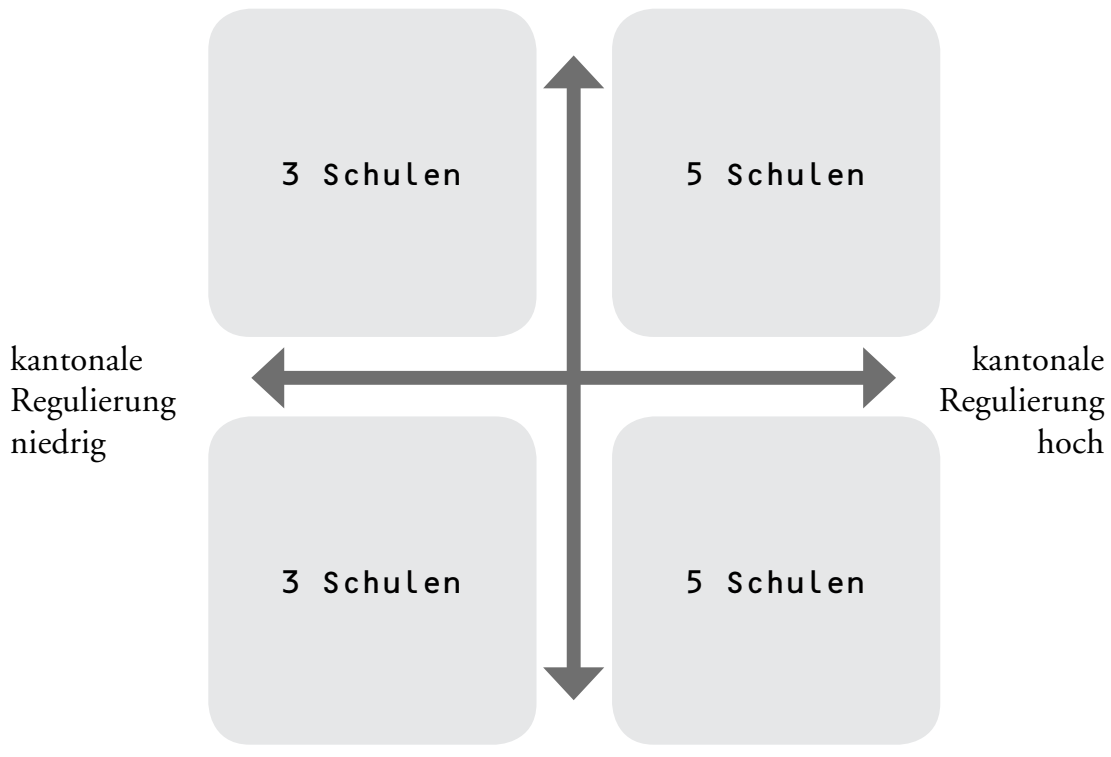

grosse Schulen

Abbildung 2: Übersicht zur Samplingstrategie

Methoden

Um verschiedene Verarbeitungsstrategien analysieren zu können, wurden vierzehn Schulen im Anschluss an eine externe Schulevaluation im Zeitraum von 2013 bis 2015 jeweils über zwölf Monate begleitet. Dabei wurden vorliegende Dokumente wie Evaluationsberichte oder Massnahmenpläne ausgewertet sowie Interviews mit verschiedenen schulischen und externen Akteuren durchgeführt. Als interne Akteure werden Mitglieder der lokalen Schulkommission, die Schulleitung und Lehrpersonen betrachtet. Die Gruppe der externen Akteure setzt sich aus der kantonalen Schulaufsicht (Inspektorat) und dem Evaluationsteam zusammen. Die Datenerhebung erfolgte bei den Einzelinterviews zu drei Zeitpunkten: Der erste Termin lag kurz nach der Rückmeldeveranstaltung der externen Evaluation, das zweite Gespräch folgte nach sechs Monaten und das letzte fand weitere sechs Monate später statt. Gruppendiskussionen mit Lehrpersonen wurden kurz nach der Rückmeldeveranstaltung geführt und nach zwölf Monaten weitergeführt.

Um das Spektrum schulischer Verarbeitungsmuster möglichst vollständig zu erfassen, wurde das Sampling mittels der zwei bereits beschriebenen Dimensionen der Schulgrösse sowie der kantonalen Regulation differenziert. Die vierzehn untersuchten Schulen verteilen sich annähernd gleichmässig auf die 
vier Felder der Samplingstrategie. Die Unterscheidung kleinerer und grösserer Schulen wird hier anhand der Hierarchiestufen der Schulleitung getroffen: Schulen, die über eine Gesamtschulleitung und ihr unterstellten Stufen- oder Schulhausleitungen verfügen, gelten als gross, während Schulen ohne differenzierte Führungsstruktur als klein gelten. Die Unterscheidung zwischen niedriger und hoher Regulation wird anhand der Verbindlichkeit von Empfehlungen und der Begleitung durch die kantonale Schulaufsicht getroffen.

Die Auswertung erfolgte anhand der qualitativen Inhaltsanalyse nach Mayring (2010). Die Interviews wurden verschriftlicht und anschliessend mit den Daten der Dokumentenerhebung im Programm Atlas.ti codiert. Als Codierungsgrundlage diente dabei der Interviewleitfaden, dessen Stichworte anschliessend am Material weiter differenziert wurden. Die Fragen dieses Leitfadens wurden aus den theoretischen Überlegungen zum Verarbeitungsprozess nach Rolff (2007) abgeleitet und konzentrierten sich auf den Verlauf der externen Evaluation, die Rezeption der Befunde und den Prozess des Generierens von Massnahmen, wobei jeweils nach der eigenen Perspektive und nach den Rollen sowie dem Verhalten anderer Akteure gefragt wurde. In einem nächsten Schritt wurde für jede Schule ein ausführliches Porträt erstellt. Die dabei entdeckten Strategien wurden in Bezug zu den Samplingmerkmalen gesetzt, um den Arbeitshypothesen nachzugehen. Es folgte eine schrittweise Verdichtung der Daten, bei der vier Verarbeitungsstrategien hervorgetreten sind, die im Folgenden dargestellt werden.

\section{Ergebnisse}

Die Analyse der Interviews folgt der Einteilung des Verarbeitungsprozesses in die Schritte des Problemnachvollzugs, der Planung der Aktivitäten und der Umsetzung (Reezigt \& Creemers, 2005; Rolff, 2007). Der Vergleich der nach diesem Muster strukturierten schulischen Fallgeschichten zeigt, dass sich die Verarbeitungsstrategien je nach Themenfeldern unterscheiden lassen. Die Themen der gewählten Massnahmen sind entweder a) vorrangig struktureller Art, b) vorrangig pädagogischer Art oder stellen c) eine Kombination von strukturellen und pädagogischen Veränderungen dar. Dabei umfassen die strukturellen Themen vornehmlich Aspekte der Organisations- und Personalentwicklung und die pädagogischen Themen unterrichtsnahe Aspekte.

Bei der Definition und Implementation von Massnahmen ist zwischen Varianten mit geringer und Varianten starker Partizipation des Kollegiums zu unterscheiden. Bei der Variante der Top-Down-Steuerung beschränkt sich diese Partizipation darauf, dass das Kollegium über die geplanten Massnahmen informiert wird. Bei der zweiten Variante ist die Entwicklung der Massnahmen in einen Dialog mit dem Kollegium eingebettet, bei der dritten Variante werden Entwürfe im Kollegium erarbeitet, sodass der Prozess im Zeichen der professio- 
nellen Mitgestaltung steht. Tabelle 1 stellt die Verteilung der Strategien innerhalb der zwei Dimensionen Themenfelder und Partizipation dar. Die Strategien A und B treten in allen vier Quadranten des Samples auf, während die Strategien C und $\mathrm{D}$ vorwiegend im Fall niedriger kantonaler Regulierung zu beobachten sind.

Tabelle 1: Verortung der Verarbeitungsstrategien

\begin{tabular}{|l|l|l|l|l|}
\cline { 3 - 5 } \multicolumn{2}{c|}{} & \multicolumn{3}{c|}{ Themenfelder } \\
\cline { 3 - 6 } \multicolumn{2}{c|}{} & $\begin{array}{c}\text { Vorranging } \\
\text { strukturell }\end{array}$ & $\begin{array}{c}\text { Strukturell und } \\
\text { pädagogisch }\end{array}$ & $\begin{array}{c}\text { Vorrangig } \\
\text { pädagogisch }\end{array}$ \\
\hline \multirow{3}{*}{ Partizipation } & Information & Strategie A & - & - \\
\cline { 2 - 6 } & Dialog & - & Strategie B & Strategie C \\
\cline { 2 - 6 } & Mitgestaltung & - & - & Strategie D \\
\hline
\end{tabular}

In Tabelle 2 wird im Hinblick auf die Entwicklungsschritte markiert, wie sich die vier Strategien unterscheiden. Die Schritte, bei denen die Lehrpersonen kaum oder gar nicht partizipieren sind mit einer Null gekennzeichnet; ihr Einbezug ist durch ein Pluszeichen symbolisiert.

Tabelle 2: Unterscheidung der Strategien anhand der Prozessschritte und der Partizipation der Lehrpersonen

\begin{tabular}{|l|c|c|c|}
\cline { 2 - 4 } \multicolumn{1}{c|}{} & Problemnachvollzug & Massnahmenplanung & Massnahmenumsetzung \\
\hline Strategie A & $\mathbf{0}$ & $\mathbf{0}$ & $\mathbf{0}$ \\
\hline Strategie B & $\mathbf{0}$ & $\mathbf{0}$ & + \\
\hline Strategie C & $\mathbf{0}$ & + & + \\
\hline Strategie D & + & + & + \\
\hline
\end{tabular}

Die Typologie der Verarbeitungsformen ist nicht normativ in dem Sinne, dass «mehr» Partizipation eine «bessere» Variante der Schulentwicklung darstellt: Stehen strukturelle Fragen im Vordergrund, kann es für die Lehrpersonen entlastend sein, wenn der Prozess der Verarbeitung der Resultate der externen Evaluation sich auf die Ebenen der strategischen und operativen Führung konzentriert. Geht es indes um pädagogische Fragen, ist der Einbezug der Lehrpersonen unabdingbar. Im Folgenden sollen die vier Verarbeitungsstrategien kurz dargestellt und anhand von einigen Zitaten illustriert werden.

\section{Strategie der Administrativen Ausführung (A)}

Der Problemnachvollzug, die Auswahl und Planung der Aktivitäten finden ausschliesslich auf den Ebenen der strategischen und operativen Führung der Schule statt. Teilweise werden die Lehrpersonen nach ihrer Sicht gefragt, es bleibt aber ungewiss, wie stark diese Stellungnahmen Berücksichtigung finden. Die Umsetzung wird entweder direkt von der Schulleitung vorgenommen oder 
als Auftrag an einzelne Personen oder eine Gruppe des Kollegiums erteilt. Die Realisierung der Massnahmen hat eher einen administrativen als einen innovativen Charakter; vorrangig geht es um die Definition und Implementation formaler Strukturen und Prozesse. Da die Themen den Unterricht der einzelnen Lehrperson wenig tangieren, nehmen diese die Massnahmenumsetzung oft als bürokratisch wahr. Das begünstigt die Einstellung, Massnahmen im Bereich der Organisationsentwicklung als Zumutung wahrzunehmen, die das pädagogische «Kerngeschäft» stören. Die Vorbehalte gegen solche Massnahmen strahlen auf die Wahrnehmung der gesamten Evaluation aus, deren Nutzen von den Lehrpersonen als gering eingeschätzt wird.

Die stark ausgeprägte hierarchische Steuerung der Strategie A wird in der folgenden Aussage einer Schulleitung deutlich:

Das Zusammenstellen von... von den Diagnoseverfahren, das ist eine ganz kurze Angelegenheit, das habe ich ins Unterrichtsteam der SHP [Schulischen Heilpädagoginnen und Heilpädagogen] gegeben und gesagt, das ist euer Job, ihr seid die Hüter davon, stellt mir das mal zusammen.... Das will ich haben (Schule I; SL_2 19) $)^{2}$.

\section{Strategie der Dialogorientierten Umsetzung (B)}

Der Problemnachvollzug, die Auswahl und Planung der Massnahmen erfolgen wiederum ausschliesslich auf den Ebenen der strategischen und operativen Führung, wobei letztere teilweise durch Steuer- oder Arbeitsgruppen in der Feinplanung unterstützt wird. Das Kollegium nimmt zu Entwürfen Stellung, wobei aber unklar bleibt, wie gross das Gewicht dieser Ansichten für den Entscheid ist. Die Massnahmenumsetzung erfolgt entweder in kleinen (Pilot-) Gruppen oder im gesamten Kollegium. Diese Umsetzung beinhaltet Aufgaben mit einer gestalterischen Komponente, bei der es darum geht, einen vorgegebenen Rahmen inhaltlich auszufüllen. Die Massnahmen beziehen sich auf formale Strukturen, die aber für den Unterricht eine wichtige Rolle spielen, wie etwa die Einführung von Unterrichtsteams oder Feedbackinstrumenten. Hinsichtlich der Effekte der Massnahmen taucht beim Typs B relativ früh die Frage auf, wie die Auswirkungen auf die Schul- und Unterrichtsqualität überprüft werden können und wie genau die Kontrollfunktion der Schulleitung aussieht.

In Fällen, bei denen einzelne Arbeits- oder Pilotgruppen die Implementation vorbereiten, kann das Problem auftreten, dass eine grössere Zahl von Lehrpersonen trotz der Anhörung des Kollegiums die Massnahmen als eher fremdbestimmt empfindet. Das folgende Zitat aus der Perspektive der Q-Gruppe verdeutlicht den Zwischenschritt, dass bei der Erstellung eines Gesamtbildes von einzelnen Rückmeldungen abstrahiert werden muss. 
... wir haben jetzt Teilziele oder, 3 Teilziele pro Unterrichtsteamgruppe erhalten, die wir erreichen müssen -- ja, bis dann. Und jetzt arbeiten wir daran, nächsten Montagnachmittag ist dann die erste Sitzung, bei der wir dann in der Gruppe daran arbeiten (Schule E; QM_3 3).

\section{Strategie der Exekutiven Mitgestaltung (C)}

Die Schulleitung studiert den Evaluationsbericht und nimmt den Problemnachvollzug vor. Daraus leitet sie Entwicklungsschwerpunkte ab, aus denen sich Aufträge an die Lehrpersonen ergeben. Diese Aufträge werden auf der Ebene des Kollegiums beraten und umfassen meist Klärungen und Standortbestimmungen mit Bezug zu den Evaluationsergebnissen. Dieser Austausch begünstigt die Entwicklung des Problembewusstseins im Kollegium. Nicht selten werden in dieser Phase weitere Daten gesammelt. Die Mitglieder des Kollegiums sind im nächsten Schritt aufgefordert, individuell oder in Gruppen Ziele abzuleiten und sich zu überlegen, wie diese zu erreichen und zu überprüfen sind. Daraus entstehen verschiedene Projekte, wobei die Schulleitung das Controlling übernimmt. Die Vorhaben beziehen sich auf unterrichtsnahe Themen wie etwa Lernziele oder gemeinsame Beurteilungsinstrumente. Dieses Vorgehen setzt entweder ein kleines Team oder aber eine Binnenstruktur von funktionierenden Jahrgangs-, Klassen- oder Unterrichtsteams voraus. Die Schulleitung ist bei der Entwicklung und der Implementation der Massnahmen vor allem in der Form indirekter Steuerung involviert und ist speziell dadurch gefordert, dass die Delegation der Entwicklungsaufgaben Eigendynamiken auslösen kann, die uneinheitliche Lösungen nach sich ziehen.

Den Stellenwert der Partizipation der Lehrpersonen bei dieser Strategie unterstreicht die folgende Aussage einer Schulleitung:

Also, bei mir war es so, dass sie es mehrmals an Konferenzen thematisiert haben. Sie haben Unterlagen gesehen. Sie haben Kurven gesehen. Sie haben pro Unterrichtsteam definiert, was geht uns was an? Wo haben wir Handlungsbedarf? Wir haben definiert: Was gibt es im Schulhaus zu verändern? (Schule J; SL_2 105).

Diese Aussage umreisst ein mehrstufiges Verfahren, bei dem der Weg vom Text zur Grafik und vom Unterrichtsteam zum gesamten Kollegium führt. Zunächst werden die Lehrpersonen in der dritten Person Plural angesprochen, bei der Implementation wechselt die Schulleitung dann aber zum «Wir» und hebt damit die Gemeinschaft der professionellen Akteure hervor.

\section{Strategie der Reflexiven Mitgestaltung (D)}

Das Kollegium ist bei der Strategie D sehr stark in die Auseinandersetzung mit den Evaluationsergebnissen einbezogen. Meist erfolgt dieser intensive Problemnachvollzug bei Evaluationsergebnissen, die erstaunen oder als diffus erscheinen. Die Daten der externen Evaluation werden akribisch interpretiert, zudem 
werden mitunter weitere Daten gesammelt. Die strategische und operative Führung strukturiert den Prozess der Definition, Auswahl und Planung von Massnahmen, das Kollegium kann aber durch Abstimmungen auf die Entscheidungen einen starken Einfluss ausüben. Die Klärung kann die Diskussion über Aspekte des schulischen Leitbildes oder über das gemeinsame Verständnis von Schul- und Unterrichtsqualität einschliessen. Der Umstand, dass pädagogische Fragen bei dieser Klärung ein grosses Gewicht haben, erschwert die Festlegung auf kontrollierbare Ziele; die Massnahmen erstrecken sich oft auf einen relativ grossen zeitlichen Horizont und bedürfen dann im Hinblick auf Kriterien oder Indikatoren noch einer weiteren Operationalisierung.

Die Klärung von Begriffen und Zuständigkeiten ist bei dieser Strategie zentral. Der folgende Interviewausschnitt verdeutlicht diesen Sachverhalt aus der Schulleitungsperspektive:

Wir haben wirklich einen halben Tag und fast einen Nachmittag zusätzlich eingesetzt, weil wir haben es offen gelassen und dann hat jeder, der ein pädagogisches Ziel will, hielt ein Kurzreferat und hatte skizziert, was das für ihn wäre. Und dort ist mehr gekommen als ich erwartet hätte. Also das war wirklich sehr spannend und ganz gute Inputs und auf Grund von dem, was dann die ganze Palette bot, hat man es reduziert und sich am Schluss demokratisch aufs Thema Differenzierung geeinigt» (Schule C; SL_1 67).

Der Verweis auf das offene Verfahren der Wortmeldung und die Entscheidung in Form einer demokratischen Abstimmung über das Thema deuten an, dass in diesem Fall die Schulentwicklung nach der externen Evaluation bottom-up eingeleitet wird.

\section{Zur Logik der Verarbeitungsstrategien}

Der Vergleich der genutzten Verarbeitungsstrategien der Schulen mit den kantonalen Rahmenbedingungen (Arbeitshypothesen 1 und 2) zeigt, dass in Systemen mit hohem Verpflichtungscharakter sämtliche Schritte des Entwicklungskreislaufes absolviert werden. Dies verwundert wenig, da die kantonale Schulaufsicht diesen Prozess begleitet und kontrolliert. Schulen in diesen Settings wählen vorwiegend Strategien der Ausführung und der aktiven Massnahmenumsetzung. Viel spricht dafür, dass die strikten Vorgaben, dazu führen, dass die Schulen eher eine instrumentelle Nutzung vorziehen und sich weniger auf ungewisse und offene Verarbeitungsstrategien einlassen. Dabei besteht die Gefahr, dass die Schulleitungen ihre Aufmerksamkeit darauf ausrichten, die einzelnen Entwicklungsschritte gegenüber der Schulaufsicht in Papierform dokumentieren zu können, ohne dass sich in der schulischen Praxis nachhaltige Veränderungen vollziehen. 
Bei den untersuchten Schulen mit niedriger kantonaler Regulierung sind alle vier Verarbeitungsstrategien zu finden. Auch hier sind Ambiguitäten im Hinblick auf die Funktionalität oder Dysfunktionalität wie auch im Hinblick auf die Implementation von Massnahmen deutlich ausgeprägt. Ob die grössere Freiheit $\mathrm{zu}$ intensiveren Anstrengungen bei der Gestaltung einer selbstbestimmten Agenda der Schulentwicklung führt, ist ungewiss. Fehlt es Schulleitungen an Durchsetzungsstärke, ist die Wahrscheinlichkeit gross, dass die Autonomie bei kritischen Befunden dazu führt, dass aus der externen Evaluation keine Konsequenzen gezogen werden.

In Bezug auf die Schulgrösse (Arbeitshypothesen 3 und 4) zeigt sich, dass der Einbezug der betroffenen Personen an kleinen Schulen einfacher möglich und zum Teil durch begrenzte personelle Ressourcen auch notwendig ist. An grösseren Schulen findet die Verarbeitung sowohl auf der Ebene der gesamten Organisation wie auch auf der Ebene einzelner Schulstufen und Schulhäuser statt. Die Lehrpersonen sind dabei nicht in den Entwicklungsprozess der Gesamtschulebene eingebunden. Diese Distanz tritt auch in den Vorbehalten gegen den Nutzen der Evaluation und die Funktionalität von Massnahmen zutage. Allerdings ist zu betonen, dass auch bei kleineren Schulen solche Vorbehalte erkennbar sind: Die Schulgrösse macht in dieser Hinsicht keinen wesentlichen Unterschied aus.

Die Darstellung der verschiedenen Verarbeitungsstrategien verweist darauf, dass die Schulen je nach Themenfeld der Massnahmen (Arbeitshypothese 5) anders verfahren. Strukturelle Elemente werden eher top-down erarbeitet; pädagogische Elemente gehen die Schulleitungen eher partizipativ an. Je stärker die Lehrpersonen einbezogen werden, desto komplexer gestaltet sich der Prozess. Strukturelle und pädagogische Themen können insofern konsekutiv geordnet werden, als die Bereinigung struktureller Probleme eine Voraussetzung dafür bildet, dass pädagogische Themen in den Vordergrund gerückt werden können.

Hinsichtlich der Nutzungsformen nach King und Pechman (1984) sowie Weiss (1998b) lassen sich die Verarbeitungsstrategien folgendermassen einteilen: Die Strategie der Administrativen Ausführung (A) ist der instrumentellen Nutzung von Evaluationsergebnissen zuzuordnen; dabei werden die Empfehlungen relativ direkt umgesetzt. Die Strategien der Dialogorientierten Umsetzung (B) und der Exekutiven Mitgestaltung (C) sind als Mischformen zwischen den Polen der instrumentellen und konzeptionellen Nutzung zu verorten.

Die Strategie der Reflexiven Mitgestaltung (D) ist der konzeptionellen Nutzung zuzuordnen, da die Evaluationsergebnisse zwar den Ausgangspunkt für die Auseinandersetzung dienen, diese Auseinandersetzung aber im Hinblick auf grundsätzliche Fragen der Schulentwicklung geführt wird. Die symbolische Nutzung von Evaluationsergebnissen kann bei allen Strategien eine Rolle spielen und tritt vor allem in der Produktion von Papieren zutage. Die Frage, ob es bei einer solchen Nutzung bleibt, ist nach der wissenschaftlichen Begleitung im Zeitraum von zwölf Monaten noch nicht abschliessend zu beantworten. 


\section{Ambiguitäten}

Die Typologie der Verarbeitungsformen hebt bestimmte Muster im Antwortverhalten hervor, sollte aber keinen falschen Anschein von Eindeutigkeit erwecken. Bei den einzelnen Fällen zeigen sich Ambiguitäten, die auf unterschiedliche Wahrnehmungen von verschiedenen Akteuren und teilweise auch auf das Schwanken einzelner Akteure hinweisen. Ambiguitäten treten einerseits bei der Frage der Funktionalität oder Dysfunktionalität der Partizipation zutage und andererseits bei der Frage nach der Konsequenz, mit der beschlossene Massnahmen in die Praxis umgesetzt werden. In beiden Fällen fungiert die Entkopplung von talk und action (Brunsson, 2002) als Entlastung.

Mitunter wählen Akteure den Modus ironischer Distanz, um anzudeuten, wie komplex die Konstellation ist:

A: Ja, also, mit dem Schulleiter zusammen. Er wird wahrscheinlich schon seine Punkte haben, die er gerne angehen würde... (Gelächter)

B: Die er uns in den Mund legen will.

A: Ja. (Spielt den Schulleiter:) Ich schlage euch vor, dass wir diese und diese Punkte angehen. Einwände? Keine, gut. (lautes Gelächter)

B: Wie sagt man, eine geleitete Demokratie, oder?» (Schule I; LP_1 256).

Der Austausch weist darauf hin, dass Partizipation unter Umständen eine Alibifunktion erfüllt. Mitsprache findet hier statt, jedoch scheinen die Ergebnisse durch hierarchische Vorgaben gezielt kanalisiert zu werden. Die rhetorische Frage nach Einwänden unterstreicht, dass es sich um einen Fall von Pseudopartizipation handeln kann. Dass die Gruppe dem Thema mit Humor begegnet, weist darauf hin, dass hier keine Böswilligkeit unterstellt wird, sondern eher ein Rollenzwang, dem die Schulleitung sich nicht entziehen kann.

Eine eher resignative Tendenz ist hingegen in dieser Äusserung erkennbar, die darauf abhebt, dass aus der Verschriftlichung von Stellungnahmen nicht automatisch Konsequenzen erwachsen:

Wir hatten eine Plenumsauswertung gemacht und das auf dem Flipchart aufgeschrieben. Was dann mit dem passiert\# Wir schreiben noch relativ viel auf solche Plakate auf (lacht).... Aber was jetzt weiter passiert, das weiss niemand (Schule I; LP_1 237).

Wenn es heisst, dass «niemand» sagen könne, was nach der Auswertung passiert, erscheint die Verarbeitung der Ergebnisse der ESE als anonymer Prozess, dem die Schulleitung ebenso unterworfen ist wie das Kollegium. Demgegenüber ist im folgenden Statement ein ausgeprägter Gestaltungswille erkennbar, bei dem der Konsens von strategischer Führung und operativer Führung betont wird, die «in erster Instanz» für den Prozess verantwortlich seien: 
Die beiden [Schulbehörde und Schulleitung] entscheiden eigentlich, was wir umsetzen. Aber ohne Lehrpersonen können wir das nicht (lacht). Das hat keinen Wert, dass wir irgendetwas machen....Und dann werden wir das mit den Lehrpersonen, sag ich jetzt mal, demokratisch entscheiden» (Schule F; SL_1 139).

Auch hier taucht der Aspekt der demokratischen Partizipation auf, wobei zu vermuten ist, dass das Kollegium über eine Vorlage abstimmt. Gleichwohl ist dieses Bekenntnis zur Demokratie doppelbödig, weil dem Kollegium das «Wir» von lokaler Behörde und Schulleitung als Einheit gegenübersteht. Die Wendung «sag ich jetzt mal» deutet darauf hin, dass sich der Schulleiter der Tatsache bewusst ist, dass das Kollegium in dieser Sache nicht das letzte Wort hat. Zugleich wird in dieser Äusserung aber auch deutlich, dass die formale Entscheidungsgewalt auf den Ebenen der strategischen und operativen Führung nicht ausreicht, um Massnahmen erfolgreich durchzusetzen.

\section{Fazit}

Im Anschluss an externe Evaluationen ergibt sich für obligatorische Schulen in der Schweiz ein gespaltenes Bild: Bei strukturellen Massnahmen dominiert die hierarchische Steuerung, während der Partizipation des Kollegiums bei pädagogischen Massnahmen ein relativ hohes Gewicht zukommt. In beiden Fällen sind Ambiguitäten feststellbar, die sich zum einen auf die Funktionalität oder Dysfunktionalität der Partizipation beziehen und zum anderen auf die Konsequenzen, die aus dem Evaluationsbericht gezogen werden. Diese Ambiguitäten können dazu führen, dass sich die Schulentwicklung im Anschluss an externe Evaluationen auf Aspekte symbolischer Nutzung beschränkt. Während bei diesen Ambiguitäten keine bedeutsamen Unterschiede im Hinblick auf die Schulgrösse feststellbar sind, verhält sich das im Hinblick auf die Regulierungstiefe anders: Bei Kantonen mit hoher Regulierung ist die Definition von Massnahmen politisch-administrativ vorgeschrieben, aber nicht immer klar, wie sich Papier und Praxis zueinander verhalten; bei Kantonen mit niedriger Regulierung beginnt die Ungewissheit an dem Punkt, ob überhaupt Massnahmen getroffen werden.

Für die Gültigkeit der Ergebnisse ist zu beachten, dass diese sich auf Selbstauskünfte einer Stichprobe von vierzehn Schulen beziehen und lediglich den Umsetzungszeitraum eines Jahres umfassen. Für weitere Studien wäre es wünschenswert, die Befunde im Rahmen eines grösseren Samples und eines grösseren Zeithorizonts zu überprüfen. Die vorliegende Analyse bestätigt die Arbeitshypothesen, dass sich die Verarbeitungsstrategien an Schulen mit hoch regulierten Vorgaben ähneln, da der schulische Autonomiespielraum stark eingeschränkt ist und dass bei Schulen im Fall der niedrigen Regulierung eine grössere 
Vielfalt an Strategien zu beobachten ist. Hingegen muss die dritte Arbeitshypothese, dass an kleinen Schulen die Partizipation der Kollegien höher sein wird als an grösseren Schulen, relativiert werden: Eine geringere Partizipation ist zwar im Austausch mit der Gesamtleitung bemerkbar, dass aber heisst nicht, dass auf den Ebenen der einzelnen Schulstufen und Schulhäuser keine Partizipation stattfindet. Die vierte Arbeitshypothese, dass sich an grossen Schulen mit hoch regulierten Evaluationssystemen die geringste Partizipation des Kollegiums zeigen wird, muss mit der Einschränkung versehen werden, dass das für strukturelle Themen gilt: Bei pädagogischen Themen wirken sich die kantonalen Rahmenbedingungen kaum auf die Partizipation des Kollegiums aus; hier dominiert die lokale Agenda der Schulentwicklung.

Bei strukturellen Themen steht die instrumentelle Nutzung der Evaluationsergebnisse im Vordergrund, bei pädagogischen Themen ergibt sich die Chance auf eine konzeptionelle Nutzung. Aufgrund der bezeichneten Ambiguitäten ist allerdings kontingent, ob es in der Tat zu einer solchen Nutzung kommt. Viel spricht dafür, dass hier die Schulleitung eine Schlüsselrolle spielt. Indes ist zu betonen, dass die konzeptionelle Nutzung von Evaluationen ebenso wenig ein Selbstwert ist wie die Partizipation bei der Ergebnisverarbeitung. Letztlich ist hier entscheidend, mit welchen Problemen die Schule konfrontiert ist.

\section{Anmerkungen}

1 Die Ergebnisse der vorliegenden Studie basieren auf Daten, die im Rahmen des Natio-nalfondsprojektes «Externe Schulevaluation und professionelle Selbststeuerung» (SNF 140739) generiert wurden.

2 Der nachfolgende Prozessschritt der Evaluation der Massnahmen wird hier nicht behandelt, da die Schulen nach dem Verlauf von zwölf Monaten noch nicht am Punkt der Überprüfung angelangt sind.

3 Die Schulen sind alphabetisch mit Buchstabenkürzeln bezeichnet worden, «SL» steht für Schulleitung, «LP» für Lehrpersonen, «QM» für Qualitätsmanagement und «Behörde» für Repräsentanten der lokalen strategischen Führung; die Zahl nach dem Unterstrich bezeichnet die Erhebungswelle.

\section{Literatur}

Altrichter, H. \& Maag Merki, K. (2010). Steuerung der Entwicklung des Schulwesens. In H. Altrichter \& K. Maag Merki (Hrsg.), Handbuch Neue Steuerung im Schulsystem (S. 15-40). Wiesbaden: Verlag für Sozialwissenschaften.

Bellmann, J., Duzevic, D., Kirchhoff, C. \& Schweizer, S. (2014). Der Sinn von Reformen und der Eigensinn der Akteure. Zur Bedeutung von Reformrezeptionstypen für Erfolg und Misserfolg Neuer Steuerung. Organisation und Verwaltung, 2, 56-59.

Bellmann, J. \& Weiss, M. (2009). Risiken und Nebenwirkungen Neuer Steuerung im Schulsystem. Theoretische Konzeptualisierung und Erklärungsmodelle. Zeitschrift für Pädagogik, 55(2), 286-308.

Böttcher, W., Dicke, J. N. \& Hogrebe, N. (2010). Einleitung. In W. Böttcher, J. N. Dicke \& N. Hogrebe (Hrsg.), Evaluation, Bildung und Gesellschaft. Steuerungsinstrumente zwischen Anspruch und Wirklichkeit (S. 9-16). Münster: Waxmann.

Böttger-Beer, M., Vaccaro, D. \& Koch, E. (2010). Wirkmodell zur externen Evaluation. In 
W. Böttcher, J. N. Dicke \& N. Hogrebe (Hrsg.), Evaluation, Bildung und Gesellschaft (S. 319-335). Münster: Waxmann.

Brunsson, N. (2002). The Organization of Hypocrisy: Talk, Decisions and Actions in Organization. Kopenhagen: Abstrakt recte.

Dalin, P., Rolff, H.-G. \& Buchen, H. (1996). Institutioneller Schulentwicklungsprozess. Bönen: Kettler.

Dedering, K. (2012). Das "Who-is-Who» der Vermessung. Friedrich Jahresheft, 40-41.

Dedering, K. \& Tillmann, K.-J. (2012). Schulqualität - Qualitätsrahmen - Schulinspektion. Friedrich Jahresheft, 44-47.

Ditton, H. (2002). Evaluation und Qualitätssicherung. In R. Tippelt (Hrsg.), Handbuch Bildungsforschung (Band 1. Auflage) (S. 775-790). Opladen: Leske und Budrich.

Ehren, M. C. M., Altrichter, H., McNamara, G. \& O’Hara, J. (2013). Impact of school inspections on improvement of schools - describing assumptions on causal mechanisms in six European countries. Educational Assessment, Evaluation and Accountability, 25(1), 3-43.

Ehren, M. C. M. \& Visscher, A.J. (2006). Towards a theory on the impact of school inspections. Britisch Journal of Educational Studies, 54(1), 12-72.

French, W. L. \& Bell, C.H. (1990). Organisationsentwicklung. Bern: Paul Haupt.

Husfeldt, V. (2011). Externe Schulevaluation. Ein länderübergreifender Blick auf Forschung und Modelle. In C. Quesel, V. Husfeldt, N. Landwehr \& P. Steiner (Hrsg.), Wirkungen und Wirksamkeit der externen Schulevaluation (S. 13-34). Bern: hep.

King, J. A. \& Pechman, M. (1984). Pinning a Wave to the Shore: Conceptualizing Evaluation Use in School Systems. Educational Evaluation and Policy Analysis, 6(3), 241-251.

Landwehr, N. (2011). Thesen zur Wirkung und Wirksameit der externen Schulevaluation. In C. Quesel, V. Husfeldt, N. Landwehr \& P. Steiner (Hrsg.), Wirkungen und Wirksamkeit der externen Schulevaluation (S. 35-70). Bern: hep.

Mahler, S. \& Quesel, C. (2015). Die externe Schulevaluation in der Schweiz. Stand und Perspektiven. Journal für Schulentwicklung, 3(19), 20-25.

Mayring, P. (2010). Qualitative Inhaltsanalyse. Grundlagen und Techniken. Weinheim: Beltz.

Pietsch, M., Van de Ham, A.-K. \& Köller, O. (2015). Wirkungen von Schulinspektion: Ein Rahmen zur theoriegeleiteten Analyse von Schulinspektionseffekten. In M. Pietsch, B. Scholand \& K. Schulte (Hrsg.), Schulinspektion in Hamburg. Der erste Zyklus 2007-2013: Grundlagen, Befunde, Perspektiven (S. 117-135). Münster: Waxman.

Quesel, C., Näpfli, J., Buser, P. \& van der Heiden, N. (2015). Bürgerbetiligung im Kontext der School Governance. Befunde einer Dokumentenanalyse zu Vorgeschichte und zur Ausgestaltung schulischer Teilautonomie in der Schweiz. In H.J. Abs, T. Brüsemeister, M. Schemmann \& J. Wissinger (Hrsg.), Governance im Bildungssystem. Analysen zur Mehrebenenperspektive, Steuerung und Koordination (S. 229-254). Wiesbaden: Springer.

Reezigt, G. J. \& Creemers, B. P. (2005). A comprehensive framework for effective school improvement. School Effectiveness and School Improvement, 16(4), 407-424.

Rolff, H.-G. (2007). Studien zu einer Theorie der Schulentwicklung. Weinheim: Beltz.

Scheerens, J. \& Bosker, R. J. (1997). The foundations of educational effectiveness. Oxford: Pergamon.

Stamm, M. (2003). Evaluation und ihre Folgen für die Bildung: eine unterschätzte pädagogische Herausforderung. Münster: Waxmann.

Verhaeghe, G., Vanhoof, J., Valcke, M. \& Van Petegem, P. (2009). Using school performance feedback: perceptions of primary school principals. School Effectiveness and School Improvement, 21(2), 167-188.

Wacker, A., Maier, U. \& Wissinger, J. (2012). Zur Bedeutung und Kritik der Steuerungsforschung - Fazit und Ausblick. In A. Wacker, U. Maier \& J. Wissinger (Hrsg.), Schul-und Unterrichtsreform durch ergebnisorientierte Steuerung (S. 303-322). Wiesbaden: Verlag für Sozialwissenschaften. 
Weiss, C. (1998a). Knowledge Creep and Decision Accretion. Knowledge: Creation, Diffusion, Utilization, 1(3), 381-404.

Weiss, C. (1998b). Have we learned anyting new about the use of evaluation? American Journal of Evaluation, 19(1), 21-33.

Wurster, S. \& Gärtner, H. (2013). Schulen im Umgang mit Schulinspektion und deren Ergebnissen. Zeitschrift für Pädagogik, 59(3), 425-445.

Schlagworte: Schulentwicklung, externe Evaluationen, Qualitätsmanagement, Governance, Partizipation

\section{Stratégies de traitement intra-scolaires des résultats des évaluations externes. Une étude comparative concernant les systèmes de scolarité obligatoire en Suisse.}

\section{Ré sumé}

Les évaluations externes doivent fournir des informations sur la qualité de l'école et contribuer à son développement. L'article porte sur la question des stratégies de traitement des résultats de l'évaluation externe au sein de l'école en se fondant sur des études de cas qualitatives dans quatre cantons suisses. L'investigation longitudinale révèle quatre stratégies qui se distinguent au niveau des pratiques de pilotage et du domaine dans lequel les mesures d'amélioration sont prises. Les résultats indiquent que le choix de la stratégie est déterminé par les thèmes centraux du rapport d'évaluation. Plus les questions relèvent de la pédagogie, plus la participation des enseignants s'avère un élément essentiel de la stratégie d'assimilation adoptée.

Mots-clés: Amélioration de la qualité scolaire, évaluation externe, gestion de la qualité, gouvernance, participation 


\section{Strategie di elaborazione dei risultati di valutazioni esterne nella scuola dell'obbligo. Un confronto fra quattro cantoni svizzeri.}

\section{Riassunto}

Le valutazioni esterne dovrebbero offrire una visione oggettiva della qualità della scuola e contribuire a migliorarne le pratiche pedagogiche e didattiche. In questo articolo ci si pone, infatti, il problema riguardo a come vengano discussi i risultati all'interno delle varie scuole, in base a uno studio qualitativo condotto in quattro cantoni svizzeri. Attraverso un'osservazione a lungo termine, è possibile riconoscere quattro strategie di elaborazione, che dipendono dai metodi di gestione per assicurare la qualità della scuola e dall'argomento delle valutazioni in funzione di tali provvedimenti. Si è così arrivati alla conclusione che le strategie di elaborazione variano a dipendenza della problematica principale della valutazione: più ci si interroga sugli aspetti pedagogici e didattici, più i docenti sono coinvolti nella discussione dei risultati.

Parole chiave: Sviluppo scolastico, valutazione esterna, certificazione della qualità, gestione, partecipazione

\section{Organisational strategies for the use of school inspection results. A comparative study on compulsory schools in Switzerland}

\section{Summary}

School inspections provide information on school quality and hints for school improvement. The article analyses organizational strategies for the use of inspection results based on qualitative case studies in four Swiss cantons. The longitudinal study reveals four organization development strategies which differ on the level of governance procedures and on the level of subject matters of change. The analysis shows that strategies depend on the focus of inspection reports. The participation of the teaching staff in organization development is considerably higher when pedagogical questions are concerned.

Keywords: School improvement, school inspection, quality management, governance, participation 
